\title{
A conceptual model described how adults responded to a simulated literacy assessment
}

\author{
Brez SM, Taylor M. Assessing literacy for patient teaching: perspectives of adults with low literacy skills.J Adv Nurs 1997 \\ May;25:1040-7.
}

\section{Objective}

To understand how adults with low literacy skills experienced an assessment of their reading ability.

\section{Design}

Multiple case study design with purposeful sampling, resulting in a conceptual model.

\section{Setting}

Community college in Ontario, Canada.

\section{Participants}

8 volunteers (age range $21-55$ y, mean age $35.6 ; 50 \%$ men) with a range of literacy skills were recruited from a community college literacy programme through self referral or referral by teachers or peers. Inclusion criteria were ability to speak English fluently, recent experience as a patient in hospital, and low literacy based on placement in a literacy programme.

\section{Methods}

Data were collected through semistructured interviews and participant observation before, during, and after a simulated literacy screening experience which used a nurse administered tool-the Rapid Estimate of Adult Literacy in Medicine. Interviews were audiotaped and transcribed verbatim. Non-verbal responses and researcher perceptions were recorded in a field log. Data were analysed using a constant comparison technique. Each case was analysed independently before cross case comparisons were done. Comparison of themes across cases provided a method of triangulation by data source.

\section{Main results}

5 interrelated themes were identified (see fig). Firstly, participants expressed fear that exposure of their low literacy would lead to reduced self esteem and social acceptance. Strategies to conceal reading limitations were common. Secondly, participants felt vulnerable while in hospital fearing that nondisclosure could affect their acquisition of knowledge normally gained from written materials, and hamper their abilities to make informed decisions and provide self care. Thirdly, fear of exposure of low literacy was moderated in the hospital context which participants viewed as a place for care, teaching, and shar-

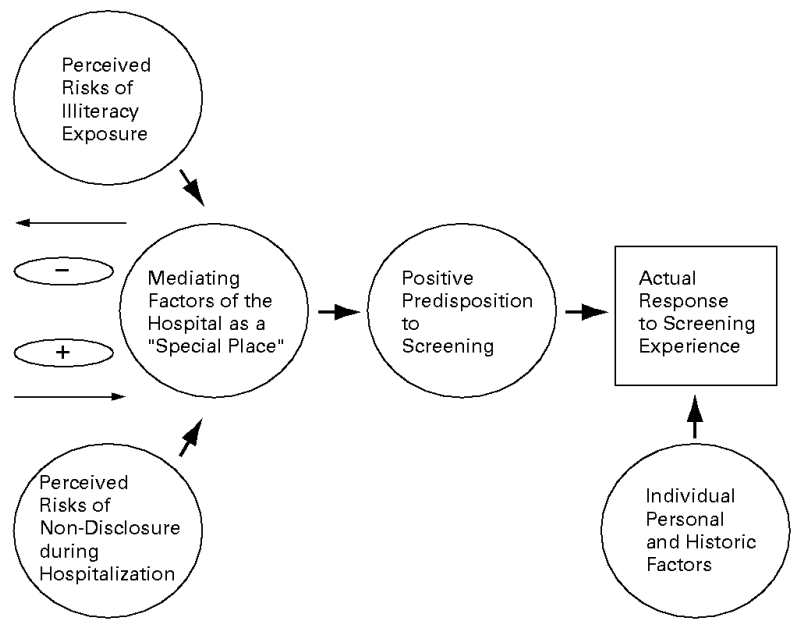

Figure A conceptual model of the response of adults with low literacy skills to literacy screening during hospitalization. Reproduced with permission from the Journal of Advanced Nursing 1997;25:1040-7.

ing of vulnerabilities. Fourthly, all participants felt that health care professionals should be aware of an individual patient's reading status, but were reluctant to volunteer this information. They wanted health care professionals to initiate discussions about reading ability. Fifthly, when participants perceived the reading assessment as a test, they responded more negatively to the experience.

\section{Conclusion}

A conceptual model of how adults with low literacy experienced literacy screening was developed, showing that responses were influenced by perceived risks of both exposure and nondisclosure of low literacy, mediated by a view of the hospital as a special place, by a positive predisposition to screening, and by personal factors which influenced the response to being assessed.

Source of funding: no external funding.

For article reprint: Ms S M Brez, Ottawa Civic Hospital, Diabetes Day Unit, 1053 Carling Avenue, Ottawa, Ontario, K1Y 4 E9 Canada.

\section{Commentary}

Nurses are often aware that literacy skills are variable within their patient population, but the use of a formal measure of reading ability is not routine in most health care settings. Interestingly, Brez and Taylor did not set out merely to assess levels of patient literacy within a specific hospital population, but chose to address the potentially more important objective of understanding how patients might feel when undertaking a test of their reading ability. The amount of written material confronting patients within the hospital environment is extensive (eg, directions, menu cards, preadmission instructions) and all assume literacy. When patients are confronted with material that they cannot read and which may be crucial for their wellbeing, the danger exists that they will not reveal their reading difficulties.

It is important for nurses to find ways to make the experience of disclosure less threatening. Respondents emphasised the importance of developing a trusting relationship with nurses to facilitate self disclosure. Nurses should initiate any dis- cussion of reading ability rather than assume the patient will offer this information voluntarily. It is also important that continued efforts ensure that written material uses clear, simple language. Information should be presented to patients using the widest possible range of options, including those that do not rely on reading ability. This could then avoid the need to implement any formal test.

Caroline Carlisle RGN, RM, MSc Head, Department of Nursing University of Liverpool Liverpool, $U K$ 